\title{
Raman Scattering from Fractals. Simulation on Large Structures by the Method of Moments
}

\author{
G.Viliani, R.Dell'Anna, O.Pilla and M.Montagna \\ INFM, Dipartimento di Fisica, Universitá di Trento, I-38050 Povo, Trento, Italy \\ G. Ruocco and G.Signorelli \\ INFM, Dipartimento di Fisica, Universitá dell'Aquila, Coppito, I-67100 L'Aquila, Italy \\ V.Mazzacurati \\ INFM, Dipartimento di Scienze e Tecnologie Biomediche e Biometria (STBB), \\ Universitá dell'Aquila, Collemaggio, I-67100 L'Aquila, Italy
}

(June 16, 2021)

\begin{abstract}
We have employed the method of spectral moments to study the density of vibrational states and the Raman coupling coefficient of large 2- and 3- dimensional percolators at threshold and at higher concentration. We first discuss the over-and under-flow problems of the procedure which arise when -like in the present case- it is necessary to calculate a few thousand moments. Then we report on the numerical results; these show that different scattering mechanisms, all a priori equally probable in real systems, produce largely different coupling coefficients with different frequency dependence. Our results are compared with existing scaling theories of Raman scattering. The situation that emerges is complex; on the one hand, there is indication that the existing theory is not satisfactory; on the other hand, the simulations above threshold show that in this case the coupling coefficients have very little resemblance,
\end{abstract}


if any, with the same quantities at threshold.

78.30.-j, 63.50.+x

Typeset using REVTEX 


\section{INTRODUCTION}

Crystalline solids are characterised by translational invariance and this symmetry property makes it possible to derive explicit analytic expressions for the vibrational eigenvectors in the harmonic approximation or, at worst, entails the diagonalization of small matrices. As a consequence, a lot is known on many physical properties that are determined by the vibrational dynamics of crystals (vibrational density of states, thermal properties, inelastic light and neutron scattering, and so on). The situation is completely different in disordered solids: in this case the lack of translational invariance often requires that the dynamical properties be studied by numerical calculations on more-or-less realistic models.

Even when the model well represents the real system, one of the factors that make a numerical calculation useful is the dimension of the sample; in many cases, the bigger the dimension, the lower the probability that finite size effects dominate the results and make any comparison with experimental data problematic.

As regards the study of the vibrational dynamics of disordered systems, and assuming that the harmonic approximation holds, one possible numerical approach is to build up the dynamical matrix and diagonalize it; this provides vibrational eigenvalues and eigenvectors, i.e. all the information that may be required. The problem is that the linear size of the matrix to be diagonalized is as large as the number of vibrational degrees of freedom, and, for example, a sample containing $10 \times 10 \times 10$ atoms would require the diagonalization of a $3000 \times 3000$ matrix: a very large matrix for not too large a system. Even though in some cases sufficient insight may be gained by considering only one degree of freedom per atom, the calculations are in general lengthy and expensive. In any case, 3-dimensional models containing, say, $20 \times 20 \times 20$ atoms are at present practically intractable with this technique.

In many cases all the detailed information contained in the eigenvalues and eigenvectors

is not necessary. For example, the density of vibrational states is a more significant quantity to compare with experiment than the sequence of eigenvalues. The important thing is that some of these relevant quantities can be calculated without diagonalizing the dynamical 
matrix, by exploiting in a different and more efficient way the information it contains.

In the present paper we will use the method of moments [1,2] to compute the density of vibrational states and the Raman coupling coefficient of model disordered systems consisting of two- and three-dimensional (hereafter 2D and 3D respectively) site and bond percolators; these were studied both at percolation threshold concentration and at higher concentration. Percolators at threshold are fractals and using arguments based on scale invariance it has been shown [3, 4 that the density of vibrational states follows a power law of the type $\rho(\omega) \propto \omega^{\bar{d}-1}$, where $\bar{d}$ is a parameter known as the spectral dimension.

In the recent literature several attempts were made to find a similar power law for the Raman coupling coefficient $C(\omega)$, which determines the (Stokes) scattered intensity in the following way:

$$
I(\omega)=(n(\omega)+1) C(\omega) \rho(\omega) / \omega
$$

where $n(\omega)$ is the Bose-Einstein population factor. As we will discuss in the following sections, the proposed scaling laws $C(\omega) \propto \omega^{x}$ are based on assumptions that are not universally accepted so that comparison with numerical simulation is of great importance. Numerical calculations of $C(\omega)$ were earlier produced for a number of percolators at threshold [5] 4] using dynamical matrix diagonalization. As mentioned, this allows for only limited dimensions of the models, and this restriction is particularly bad in this case, since there are indications [7,8] that power laws for $C(\omega)$ might apply only at low frequency. In the present paper we present an exhaustive analysis of $C(\omega)$ in much larger systems, in order to avoid as far as possible finite size effects and reach the lowest possible frequency.

In section II we summarize the method of calculation; section III is devoted to the stability and precision problems of the procedure and to the way we were able to overcome over- and under-flow difficulties; in section IV we present the numerical data on various types of percolators; the data are discussed in connection with the phenomenology of Raman scattering from real disordered solids in section $\mathrm{V}$. 


\section{THE METHOD OF MOMENTS}

In the following we shall outline the method of spectral moments, which is reported in detail in the papers by Benoit and coworkers [1]. This method applies to the case where one is interested in calculating a spectral function $f\left(\omega^{2}\right)$ of the form:

$$
f\left(\omega^{2}\right)=\sum_{\lambda=1}^{3 N} d_{\lambda}^{2} \delta\left(\omega^{2}-\omega_{\lambda}^{2}\right)
$$

where

$$
d_{\lambda}=\sum_{i=1}^{3 N} p_{i} e_{i}(\lambda)
$$

The system consists of $\mathrm{N}$ identical masses which interact via identical harmonic potentials and is characterized by the $3 N \times 3 N$ dynamical matrix $\mathbf{D}$ with eigenvalues $\omega_{\lambda}$ and eigenvectors $e_{i}(\lambda) ; \lambda=1 \ldots 3 N$ labels the normal modes and $i$ is a collective index that labels the masses $(l=1 \ldots N)$ and the cartesian components $(\alpha=x, y, z)$ of the eigenvectors, $i=(l, \alpha)$. The coefficients $p_{i}$ depend on the spectral function to be computed; the explicit expressions of $p_{i}$ for the cases of interest in this paper are discussed in the Appendix.

It has been shown by Benoit and coworkers [1] that $f\left(\omega^{2}\right)$ can be put in the form

$$
f\left(\omega^{2}\right)=-\frac{1}{\pi} \lim _{\varepsilon \rightarrow 0^{+}} \operatorname{Im}\{\mathrm{R}(\mathrm{z})\}
$$

where $z=\omega^{2}+i \varepsilon$, and

$$
R(z)=\int_{-\infty}^{+\infty} \frac{f\left(\omega^{2}\right)}{z-\omega^{2}} d \omega^{2}
$$

The latter can be developed as a continuous fraction

$$
R(z)=\frac{1}{z-a_{1}-\frac{b_{1}}{z-a_{2}-\frac{b_{2}}{z-a_{3}-\ldots \ldots}}}
$$

where the real coefficients $a_{n}$ and $b_{n}$ depend on the generalized moments $\nu_{n m}$ and $\bar{\nu}_{n m}$ of $f\left(\omega^{2}\right)$ (whence the name of the method):

$$
a_{n+1}=\frac{\bar{\nu}_{n n}}{\nu_{n n}}, \quad b_{n}=\frac{\nu_{n n}}{\nu_{n-1, n-1}}
$$


where

$$
\nu_{n n}=\int_{0}^{1} P_{n}\left(\omega^{2}\right) P_{n}\left(\omega^{2}\right) f\left(\omega^{2}\right) d \omega^{2}
$$

and

$$
\bar{\nu}_{n n}=\int_{0}^{1} P_{n}\left(\omega^{2}\right) P_{n}\left(\omega^{2}\right) f\left(\omega^{2}\right) \omega^{2} d \omega^{2}
$$

$\left\{P_{n}\left(\omega^{2}\right)\right\}$ is the succession of polynomials orthogonal with respect to the spectral function $f\left(\omega^{2}\right)$ (as in the rest of this paper, the frequency scale in equations 7 and 8 has been normalized so that the maximum frequency is 1$)$. The moments in turn can be computed by a recursive procedure that makes use of the dynamical matrix. Let $\mathbf{t}^{0}$ be the normalized vector with components proportional to $p_{i}$, it turns out that [1]

$$
\nu_{n n}=\left(\mathbf{t}^{(n)}, \mathbf{t}^{(n)}\right), \quad \bar{\nu}_{n n}=\left(\mathbf{t}^{(n)}, \mathbf{D t}^{(n)}\right)
$$

where $\mathbf{t}^{(n)}$ is a vector which obeys the recursive relation

$$
\mathbf{t}^{(n+1)}=\left(\mathbf{D}-a_{n+1}\right) \mathbf{t}^{(n)}-b_{n} \mathbf{t}^{(n-1)}
$$

Equations 6 to 10, with $\mathbf{t}^{(-1)}=0$, determine $a_{n}$ and $b_{n}$ recursively, and these give $f\left(\omega^{2}\right)$ through $R(z)$.

In principle, if one computed a number of $a_{n}$ and $b_{n}$ coefficients equal to the number of distinct eigenvalues [1], in the limit $\varepsilon \rightarrow 0$ one would reproduce exactly the response function in Eq. (4).

In computations of practical interest (i.e. on systems with a large number of masses) this is neither possible nor necessary. The number of moments to be computed and the value of $\varepsilon$ depend on the frequency range where the spectral function is to be reproduced most carefully.

The use a finite value for $\varepsilon$, which is equivalent to considering the convolution of $f\left(\omega^{2}\right)$ with a lorentzian

$$
L_{\varepsilon}\left(\omega^{2}\right)=\frac{\varepsilon}{\pi\left(\varepsilon^{2}+\omega^{4}\right)}
$$


makes it impossible to resolve spectral features with width of the order of (or smaller than) $\varepsilon$.

The fact that only a limited number of moments is computed introduces a truncation error that, as noted by Turchi et al. [9], can be minimized when the spectral function $f\left(\omega^{2}\right)$ has no gaps and no divergences. In this case the coefficients $a_{n}$ and $b_{n}$ tend to make small fluctuations about constant asymptotic values as $n \rightarrow \infty$, and this suggests to substitute the asymptotic values in Eq. (5) for the coefficients that are not computed. If one wants only $M$ moments one re-writes Eq. (5) as:

$$
R(z)=\frac{1}{z-a_{1}-\frac{b_{1}}{z-a_{2} \cdots-\frac{b_{M-1}}{z-a_{M}-T_{M}(z)}}} .
$$

and under the assumption of constant $a_{n}=a$ and $b_{n}=b$ for $n \geq M, T_{M}(z)$ is easily found to obey

$$
T_{M}(z)=\frac{1}{z-a-b T_{M}(z)}
$$

Since in our case $a_{n}$ and $b_{n}$ continue to make small oscillations about $a$ and $b$ respectively for large $n$, this procedure provides only an approximation to the true $R(z)$; in any case $T_{M}(z)$ becomes less and less important as the number of moments that are actually computed increases.

\section{STABILITY AND PRECISION OF THE PROCEDURE}

Since we will be mainly interested in studying the behavior of spectral functions like in Eq. (2) at frequencies of the order of $10^{-3} \div 10^{-2}$ times the Debye frequency, the use of many (some thousand) moments is necessary, as can be seen empirically and deduced theoretically. This requires many iterations of Eq. (10) that can introduce numerical errors in the computed $f\left(\omega^{2}\right)$. The errors can arise both from the precision of the calculation (accumulation of roundoff approximations) and/or from the instability of the algorithm, i.e. 
from the sensitivity of the algorithm to small random departures of $a_{n}$ and $b_{n}$ from the true values.

As a first example in Fig. 1 we show the effect of the number of computed moments on the density of states of $2 \mathrm{D}, 650 \times 650$ site percolators at percolation threshold, consisting of identical masses connected by identical springs; each mass has only one degree of freedom, cyclical boundary conditions are imposed [10]. It is clearly seen that in passing from 50 to 5000 iterations the minimum frequency at which the expected density of states $\left(\rho(\omega) \propto \omega^{0.31}\right)$ 111 is reproduced, decreases by more than one order of magnitude. Note that 1000 iterations are not sufficient. The origin of the dip at $\omega \approx 0.7$ will be discussed later.

The necessity of so many iterations may be justified theoretically as follows. Using the orthogonality relation

$$
\int_{0}^{1} P_{n}\left(\omega^{2}\right) \omega^{2 k} f\left(\omega^{2}\right) d \omega^{2}=0
$$

with $k=0, \ldots, n-1$, equation (7) becomes:

$$
\nu_{n n}=\int_{0}^{1} P_{n}\left(\omega^{2}\right)\left(\omega^{2}-1 / 2\right)^{n} f\left(\omega^{2}\right) d \omega^{2}
$$

from which it is easily seen that, for large $n, \nu_{n n}$ is mostly determined by the extremes of the $\omega$ range, so that the new information that is added at each step is more and more concerned with the extremes; the new information is necessary to approach the minimum attainable frequency, as seen in Fig. 1 [12]. Moreover, it is also clear that in order to reproduce the behaviour of $f\left(\omega^{2}\right)$ in the central region of frequencies it is neither necessary nor (as we will see) advisable to use many iterations.

The first numerical difficulty that is met in the calculation of many moments is that $\nu_{n n}$ (and $\bar{\nu}_{n n}$ ) tend to approach 0 or $\infty$, causing overflow or underflow of the computed moments. This difficulty can be overcome by noting [1] that if one scales the physical characteristics of the system (masses and/or elastic constants) by a factor $s$, the generalized moments scale as well:

$$
\mathcal{K} \rightarrow s \mathcal{K}
$$




$$
\begin{aligned}
\nu_{n n} & \rightarrow s^{2 n} \nu_{n n} \\
\bar{\nu}_{n n} & \rightarrow s^{2 n+1} \bar{\nu}_{n n} \\
b_{n} & \rightarrow s^{2} b_{n} \\
a_{n} & \rightarrow s^{2} a_{n}
\end{aligned}
$$

It is therefore possible to choose a value of $s$ such that the generalized moments are finite. Since $b_{n}=\nu_{n n} / \nu_{n-1, n-1}$, one possibility is to start with an arbitrary elastic constant $\mathcal{K}_{1}$, then compute a sequence of $M_{1}$ coefficients $b_{n}$ until $\nu_{M_{1} M_{1}}$ reaches the over/under-flow condition, then compute $\langle b\rangle=M_{1}^{-1} \sum_{n} b_{n}$ and scale $\mathcal{K}_{1}$ in agreement with the first of equations 11 so that $\langle b\rangle$ is equal to a desired value.

At first sight, it would seem that $\langle b\rangle=1$ is a good choice to avoid divergences, so that for the scaling factor $s$ we have

$$
s^{2}=\frac{1}{\langle b\rangle}
$$

This procedure may be repeated several times, until the desired number of moments can be calculated.

However $\langle b\rangle=1$ is not the most convenient choice. In fact, since the $b_{n}$ 's fluctuate around $\langle b\rangle$, we can put $b_{n}=\langle b\rangle\left(1+\varepsilon_{n}\right)$, with $\left\langle\varepsilon_{n}\right\rangle=0$; in this way we have

$$
\nu_{n n}=\langle b\rangle\left(1+\varepsilon_{n}\right) \nu_{n-1, n-1}
$$

or

$$
\begin{aligned}
\nu_{n n} & =\langle b\rangle^{n} \nu_{00} \prod_{i=1}^{n}\left(1+\varepsilon_{i}\right) \\
& =\langle b\rangle^{n} \nu_{00}\left(1+\sum_{i} \varepsilon_{i}+\sum_{i \neq j} \varepsilon_{i} \varepsilon_{j}+\ldots\right)
\end{aligned}
$$

Since $\sum_{i} \varepsilon_{i}=0$, we have

$$
\sum_{i \neq j} \varepsilon_{i} \varepsilon_{j}=\sum_{i j} \varepsilon_{i} \varepsilon_{j}-\sum_{i} \varepsilon_{i}^{2}=-\sum_{i} \varepsilon_{i}^{2}=-n\left\langle\varepsilon^{2}\right\rangle
$$

so that

$$
\nu_{n n} \approx \nu_{00}\langle b\rangle^{n}\left(1-n\left\langle\varepsilon^{2}\right\rangle\right) \approx \nu_{00}\langle b\rangle^{n} e^{-n\left\langle\varepsilon^{2}\right\rangle}
$$


Thus, in order to control the divergence of $\nu_{n n}$ one should not impose $\langle b\rangle=1$, but

$$
\langle b\rangle=e^{\left\langle\varepsilon^{2}\right\rangle}
$$

We have verified that with this choice the number of corrections of the elastic constant necessary for all the 5001 iterations of a medium-sized system (square lattice of linear size 100), or for 5000 iterations of a larger system (2D percolator of linear size 650), is smaller than with the first choice.

However, neither correction procedure eliminates a further problem concerning the orthogonality of the series $\left\{\mathbf{t}^{(n)}\right\}$. In fact, from Eq. (10) it is easy to see that $\left(\mathbf{t}^{(n)}, \mathbf{t}^{(m)}\right) \propto \delta_{n m}$, but in practical calculations the orthogonality is lost if $|n-m|$ is large enough.

In Fig. 2 we report $\left(\mathbf{t}^{(0)}, \mathbf{t}^{(n)}\right)$ as a function of $n$ for four different systems: full (ordered) square lattices of linear sizes 50 and 100, and site percolators at threshold concentration of linear sizes 50 and 650. The calculations were carried out in double precision, but using single precision only increases the value of the initial plateau. It is interesting to note that the value of $n$ at which orthogonality begins to fade does not depend in a simple way on the number of masses (and therefore on the number of required computer operations): in fact, for the larger ordered lattice orthogonality is maintained for a greater number of iterations, while for percolators this effect is much less evident, if any.

This behaviour can be understood by noting that the recursive relation 10 is very similar to the one used in the Lanczos procedure of tridiagonalization, and in fact the two become identical if in the Lanczos procedure [13] one makes the substitutions:

$$
\begin{aligned}
\mathbf{r}_{j} & \rightarrow \mathbf{t}_{j} / \sqrt{\mathbf{t}_{j} \cdot \mathbf{t}_{j}} \\
\alpha & \rightarrow a \\
\beta^{2} & \rightarrow b
\end{aligned}
$$

As discussed at length in reference [13], this loss of orthogonality is inherent and not caused by accumulation of numerical roundoff errors. Moreover, it is known that the Lanczos pro- 
cedure without any re-orthogonalization works well for the extreme eigenvalues and eigenvectors, while there are problems for the inner range.

From another point of view, the iterative equation (10) may be considered as a non linear logistic application (the non linearity coming from the dependence of $a_{n}$ and $b_{n}$ on $\mathbf{t}^{(n)}$ ) in an $N$-dimensional space. It is known that these equations admit chaotic solutions for the $\mathbf{t}^{(n)}$ variables, i.e. they produce sequences $\left\{\mathbf{t}^{(n)}\right\}$ that, as $n$ grows, become very unstable with respect to little variations of $\mathbf{t}^{(0)}$. Therefore, for example, from $\mathbf{t}^{(0)}$ numerically we obtain $\mathbf{t}^{\prime(1)}=\mathbf{t}^{(1)}+\delta \mathbf{t}^{(1)}, \delta \mathbf{t}^{(1)}$ being the machine precision error. Consequently, because of the intrinsic chaoticity of equation (10), the computed $\left\{\mathbf{t}^{\prime(n)}\right\}$ sequence will diverge from the "true" one and $\left(\mathbf{t}^{(0)}, \mathbf{t}^{\prime(n)}\right) \neq 0$.

Our numerical data are in complete agreement with the above results of matrix calculus. First of all, when we compute the density of states (or the Raman coupling coefficient) of a linear chain, orthogonality is preserved no matter how long the chain; this is expected because in this case the dynamical matrix is tridiagonal.

Moreover, when comparing the outcome of the method of moments with the eigenvalues of an exactly solvable model (square harmonic lattice with identical masses and springs with fixed boundary conditions [14]) we find that as orthogonality begins to be lost the peaks of the density of states no longer fit the exact eigenvalues in the central part of the (linear) spectrum, while the extreme eigenvalues are still well fitted, provided we use a sufficient number of moments. In any case, the central part of the spectrum is perfectly fitted only for systems small enough that computation of all moments does not result in non-orthogonal vectors. For larger systems, increasing the number of moments worsens the look of the central part of the spectrum, be it the density of states or the Raman coupling coefficient, and this is the origin of the dip in the density of states of Fig. 1. In our case, this is not a serious problem. In fact, on the one hand we are mostly interested in the low frequency part of the coupling coefficients, this being the very reason why we compute so many moments; on the other hand, if we should be interested in the central range we would compute only a few moments. This is possible because the coupling coefficients are smooth functions due to 
disorder, we don't need to resolve one peak from the next, all we need is a frequency-averaged result.

\section{NUMERICAL RESULTS ON RAMAN COUPLING COEFFICIENTS}

We proceed now to presenting the numerical data. As mentioned in the introduction, the problem of finding a scaling law for the Raman coupling coefficient in fractals, if it exists, has been the object of several experimental and theoretical papers [7, 15, 19]. Besides being interesting on their own, fractals are thought to be reasonably representative and relatively simple models of important classes of disordered systems, especially as regards vibrational characteristics. Raman scattering is one of the most convenient experimental techniques to study vibrations in disordered solids: it does not require big facilities and, contrary to the case of crystals, all vibrational modes contribute to the scattering in a disordered system. The price to be paid is that $C(\omega)$ in equation (1) is not known a priori, so that, as mentioned, an important question regards whether or not it can be cast in the form $C(\omega) \propto \omega^{x}$, and what expression $x$ has in terms of the spectral dimension $\bar{d}$, of the fractal dimension $D$, and possibly of other parameters which characterize the fractal.

All the expressions proposed for $x$ so far were derived under the assumption that $C(\omega)$ can be determined by considering the scaling properties of the strain induced by the vibrations of the fractal (the so-called fractons) [7, 15, 16, 18, 22]. In two recent papers [5,6] we argued against such possibility on the basis of numerical calculations of $C(\omega)$ in $2 \mathrm{D}$ and $3 \mathrm{D}$ site percolators, which apparently showed that $(i)$ none of the proposed $x$ could fit the numerical data in 2D, and $(i i)$ a single $x$ seems not to be sufficient in 3D for the Dipole- Induced-Dipole (DID) scattering mechanism [6] (see Appendix).

The conclusion we arrived at on the basis of these numerical results, i.e. that the existence of scaling for $C(\omega)$ is not always evident in fractals, has been recently challenged on the basis of the following arguments: $(i)$ the size of the percolation clusters employed $(60 \times 60$ in $2 \mathrm{D}$, $29 \times 29 \times 29$ in 3 D) was too small to allow positive conclusions to be drawn on scaling, which 
is expected to hold at low frequency [8], and (ii) even more so for site-percolators: bondpercolators should be used instead, because for the latter the scaling regime starts at higher frequency [7].

These issues will be discussed in the following, after we have reported numerical data obtained with the method of moments for a variety of much larger systems including site- and bond-percolators at and above percolation threshold, whose scattering units may or may not have random electrical polarizabilities (electrical disorder), and assuming different scattering mechanisms: DID, DID truncated to nearest neighbors (NNDID), and Bond-Polarizability (BPOL).

In Fig. 3 we report $C(\omega)$ for the DID and BPOL scattering mechanisms for $650 \times 650$ $2 \mathrm{D}$ site percolators at threshold. The interesting features in this figure are: (i) The BPOL spectrum shows a single slope, $m=1.24$, down to the minimum frequency, (ii) the DID spectrum clearly shows a crossover at $\omega \approx 8 \times 10^{-3}$ from $m \approx 0.94$ (high frequency) to $m \approx 0.8$, which was uncovered in [5,6] due to limited size and statistics.

In Fig. 4 we show the same coupling coefficients for 3D site percolators of linear size 80. In agreement with the results of diagonalization, [5,23] there is a very evident crossover in the DID case, while the BPOL spectrum again is a straight line in almost the whole frequency range. The open circles represent the DID spectrum of clusters of linear size 40. Apart from the obvious fact that the minimum frequency is greater in this case, we do not observe any significant difference with respect to the larger clusters, indicating that, at least for $C(\omega)$, finite size effects play a minor role, if any. It is interesting to mention that removal of dangling bonds [25] in the site percolators does not change the look and the slope of the BPOL coupling coefficients both in 2D and 3D, but it does change the density of states which does not appear to follow an $\omega^{x}$ law in the whole frequency range; this effect is particularly evident in $3 \mathrm{D}$.

The $C(\omega)$ 's relative to 2D (linear dimension 500) and 3D (linear dimension 70) 24] bond percolators are reported in Figs. 5 and 6 respectively, for both scattering mechanisms DID and BPOL. The BPOL spectra are well fitted by straight lines with almost the same slopes 
$\left(m_{2 D}=1.26, m_{3 D}=1.58\right)$ as for site percolators. The DID spectra have a roundish look, but in the low frequency part they are reasonably fitted by straight lines.

For bond percolators, The nearest-neighbor DID coupling coefficients in 2D and 3D are practically identical to DID, in agreement with what found in ref. [7], and we will not report them. This fact can be easily understood considering that in bond percolators (contrary to site percolators) it is possible to find nearest neighbor sites which are not connected by any bond. This results in highly uncorrelated motions of nearby masses that produce most of the DID scattering.

In Fig. 7 we show the effect of the so called electrical disorder on the DID scattering of $2 \mathrm{D}$ site percolators. Each mass is randomly assigned one of two values $\alpha_{1}$ or $\alpha_{2}$ of the bare polarizability; the curve of Fig. $3\left(\alpha_{1}=\alpha_{2}=1\right)$ is also reported for comparison. Note that spectra with different electrical disorders coalesce at low frequency $\left(\omega<2 \times 10^{-2}\right)$. Qualitatively the same result is obtained with all other types of clusters, with and without dangling bonds. In the case of BPOL for $2 \mathrm{D}$ site percolators the $C(\omega)$ 's with electrical disorder show a change of slope at $\omega \approx 10^{-2}$.

In Fig. 8 are reported the DID and BPOL spectra for 3D site percolators having a concentration $(c=0.5)$ higher than the percolation threshold concentration, together with the relative density of states. The latter exhibits the well known [26] crossover at $\omega_{\rho} \approx$ $7 \times 10^{-2}$ with change of slope. The fact that neither slope is equal to what expected for phonons $(m=2)$ or fractons $(m=0.31)$ is also known [26]. The $C(\omega)$ 's also change their frequency dependence at a frequency, $\omega_{C} \approx 6 \times 10^{-2}$, which is apparently slightly lower than $\omega_{\rho}$. The spectra are rather noisy and it is impossible to judge whether $C(\omega)$ is a straight line at low frequency, but in any case it is clear that below $\omega_{C}$ the "slopes" are very different from what was found for the same percolators at threshold, see Fig. 4. The same qualitative behavior is observed in 2D, and for bond percolators, though the $3 \mathrm{D}$ spectra in this case are even more noisy at low frequency.

The slopes observed are summarized in Table I for the different kinds of percolators and scattering mechanisms considered; in the case of DID, where different slopes ore found in 
the same spectrum, we report the low frequency ones for the reason discussed in the next section.

\section{DISCUSSION}

From the results presented in the previous section, it appears that $C(\omega)$ behaves quite differently depending on the scattering mechanism. In fact, in the case of BPOL the numerically computed coefficients follow very closely the law $C(\omega) \propto \omega^{x}$ almost in the whole investigated frequency range; the values of $x$ do not depend appreciably on site- or bondpercolation, nor on the presence or absence of dangling bonds. The situation is rather different for DID scattering; with this mechanism, in none of the case studied does $C(\omega) \propto \omega^{x}$ hold in the whole range of frequencies. Following the arguments of refs. [7, [8], we shall assume that it is the low frequency part of the DID spectra that is to be compared with the proposed scaling laws (but it is not clear to us why the arguments of those references do not apply to BPOL). This is supported by the result of Fig. 7, that shows that the effect of electrical disorder, which is a local random disturbance and as such is not expected to scale, disappears at more-or-less the same frequency $\left(\omega \approx 10^{-2}\right)$ where $C(\omega)$ changes slope.

Since the DID and BPOL coupling coefficients are so different and produce different slopes, we can compare our results only with the theoretical model of Alexander et al [19] because to our knowledge this is the only paper where different scattering mechanisms are considered. Alexander et al consider DID and NNDID: the latter coincides with BPOL for site percolators and, as mentioned, it is practically identical to DID for bond percolators, so that it is possible to compare our numerical data with the formulas of ref. [19] that are:

$$
C(\omega)_{D I D} \propto \omega^{(2 \bar{d} / D)(\sigma+d)-3 \bar{d}}
$$

and

$$
C(\omega)_{N N D I D} \propto \omega^{2 \bar{d} \sigma / D}
$$


where $\sigma$ is a scaling index introduced by Alexander et al [19] whose value is not known $a$ priori: for a homogeneous medium $\sigma=1$, while $\sigma<1$ means a violation of scaling [19]. Values of $\sigma \approx 1$ were deduced in ref. [8] on the basis of simulations [7] performed on smaller clusters than done here. Equation (16) was first derived by Boukenter et al [15].

Fitting the slopes with equations (15) and (16) we obtain the values of $\sigma$ reported in Table I. The picture that emerges is complex.

- (i) In two cases, 3D site percolators (DID) and 2D bond percolators (DID) we find $\sigma \approx 1$, which is the desired value;

- (ii) in two cases, 2D site percolators (NNDID) and 3D bond percolators (DID), the values found are smaller than 1 , but not too much $(\sigma \approx 0.9)$;

- (iii) in the remaining four cases, $\sigma$ is either too large to be plausible $(\sigma \approx 1.4$ for $2 \mathrm{D}$ site percolators (DID) and 3D site percolators (NNDID)) or exceedingly small $(\sigma \approx 0.3$ for 2D and 3D bond percolators (NNDID)).

The first thing that we note is that so far $\sigma$ has always been thought of as a parameter that characterizes the scattering system, not the scattering mechanism, so that the wide variations observed in Table I between DID and NNDID apparently imply that equations (15) and/or (16) are not right.

On the other hand, the fact that the BPOL $C(\omega)$ 's are straight lines over a frequency range of more than two orders of magnitude, and are so insensitive to the nature of the system, might reasonably be taken as indication that for this scattering mechanism scaling holds, but following a law other than equation (16) for site percolators. In our opinion it would not be too much of a surprise if BPOL should scale and DID should not because the modulation of polarization in DID proceeds through electromagnetism, which definitely does not propagate along the fractal paths. In any case, we think that nothing should be taken for granted when treating this subject. For example, as mentioned in the previous section, removal of dangling bonds from 2D and (especially) 3D site percolators produces 
a situation where the mass distribution follows the same power law as in the case with dangling bonds, $C_{B P O L}(\omega)$ does the same, but the density of states is no longer a power law for $\omega>\approx 4 \times 10^{-2}$. This situation might even be interpreted as indication that BPOL's being a straight line has actually nothing to do with dynamic scaling: in fact, in these systems $C_{B P O L}(\omega)$ is a straight line in a frequency region where the density of states (i.e. the most believed scaling dynamical quantity) is not. At present we are not able to resolve this question.

Another result in Table I that is worth a comment is the value of $\sigma$ for $2 \mathrm{D}$ and $3 \mathrm{D}$ bond percolators under the NNDID scattering mechanism. The fact that for these percolators DID and NNDID $C(\omega)$ 's are almost identical implies that at all frequencies available to the present simulation the scattering coefficient is mostly determined by pairs of nearest neighbor masses that are not connected by a bond. This situation produces $\sigma \approx 0.3$ for NNDID. In principle, however, it cannot be excluded that on much larger clusters the contribution of these pairs may become less important. In order to find $\sigma \approx 1$ [27] the simulation should yield NNDID slopes of $\approx 1.1(3 \mathrm{D})$ and $\approx 1.4(2 \mathrm{D})$, i.e. very different from the values obtained here. In any case, these new slopes would be found in a frequency range $\left(\omega \sim<10^{-4}\right)$ so low to be of little physical significance.

This remark introduces another important issue, i.e. the possibility of extracting from experimental spectra information on the static and dynamic fractal parameters. This is in general done by fitting the low-frequency parts of the spectra (where scaling is expected to hold) with expressions like Eqs. (15) or (16). Assuming that one has the right equations, one obvious shortcoming of this procedure is that one should know the scattering mechanism (DID, BPOL, NNDID, a mixture, ...), which has been shown to be so important in determining the low frequency slope. But even if this was known, there is another problem that is evident from fig. 8. In fact, real disordered materials cannot reasonably be thought of as percolators at threshold: if the percolation model is to have a sense, it must be a percolator above threshold. From fig. 8 we see that in this case the values of the "slopes" bear no resemblance with those found at threshold, and especially so at low frequency. This 
fact has long been known as regards the density of states of percolators above threshold [26]. Of course, the particular concentration value used here $(c=0.5)$ has no special meaning, nor is it thought to represent any particular physical system.

In conclusion, we think that the search for the scaling laws which might possibly govern Raman scattering from fractal objects is interesting in itself and as such worth being pursued; on the basis of the present numerical simulations, we think that these laws have not been found yet. Even more disputable is the extraction of fractal parameters from the experimental Raman spectra. In this regard, we frankly hope that the present paper may encourage a realistic reconsideration of previous work, even if this might lower the role of the fractal model of disordered solids.

\section{ACKNOWLEDGMENTS}

We are indebted to M. Sampoli and L. Sampoli for useful discussions and for bringing to our attention the similarities between the method of moments and the Lanczos procedure.

\section{APPENDIX: DEFINITION OF THE INITIAL VECTORS}

The general expression for the Raman intensity in $\alpha, \beta$ polarization at energy $\hbar \omega$ and exchanged momentum $\hbar \mathbf{k}$ is given by:

$$
I_{\alpha \beta}(\omega)=\mathcal{A} \int d t e^{i \omega t} \sum_{l l^{\prime}}\left\langle\pi_{\alpha \beta}^{l}(t) \pi_{\alpha \beta}^{l^{\prime}}(0) e^{i \mathbf{k} \cdot\left(\mathbf{R}_{l}(t)-\mathbf{R}_{l^{\prime}}(0)\right)}\right\rangle
$$

where $\mathcal{A}$ is a constant and $\pi_{\alpha \beta}^{l}(t)$ is the $\alpha, \beta$ component of the effective polarizability tensor of the $l$-th atom placed at site $\mathbf{R}_{l}(t)$. The time dependence of this tensor is caused by the relative displacement of atom $l$ with respect to all other atoms.

The instantaneous position $\mathbf{R}_{l}(t)$ may be written as

$$
\mathbf{R}_{l}(t)=\mathbf{x}_{l}+\mathbf{u}_{l}(t)
$$


where $\mathbf{u}_{l}(t)$ is the displacement from the equilibrium position $\mathbf{x}_{l}$. For small displacements, both the effective polarizability and the exponential function in equation (A1) can be expanded in power series:

$$
\begin{aligned}
& e^{i \mathbf{k} \cdot \mathbf{R}_{l}(t)} \approx e^{i \mathbf{k} \cdot \mathbf{x}_{l}}\left(1+i \mathbf{k} \cdot \mathbf{u}_{l}(t)\right) \\
& \pi_{\alpha \beta}^{l}(t) \approx \pi_{\alpha \beta}^{l}+\sum_{m} \sum_{\gamma} \frac{\partial \pi_{\alpha \beta}^{l}}{\partial u_{m \gamma}} u_{m \gamma}(t) .
\end{aligned}
$$

The displacement, in turn, may be expanded in normal modes:

$$
u_{m \gamma}(t)=\sqrt{\frac{\hbar}{2 M N}} \sum_{\lambda} \frac{1}{\sqrt{\omega_{\lambda}}} e_{m \gamma}(\lambda) A_{\lambda}(t)
$$

where $A_{\lambda}(t)$ are the normal coordinates that (for the Stokes part of the spectrum) obey the relation:

$$
\int d t e^{i \omega t}\left\langle A_{\lambda}(t) A_{\lambda^{\prime}}(0)\right\rangle=\delta_{\lambda \lambda^{\prime}} \frac{[n(\omega)+1]}{\omega} \delta\left(\omega-\omega_{\lambda}\right)
$$

From the previous equation we have

$$
I(\omega)=\mathcal{A} \frac{[n(\omega)+1]}{\omega} \rho(\omega) C(\omega)
$$

having defined

$$
\begin{gathered}
C(\omega)=\sum_{\lambda}\left|C_{\lambda}\right|^{2} \delta\left(\omega-\omega_{\lambda}\right) / \sum_{\lambda} \delta\left(\omega-\omega_{\lambda}\right) \\
=\sum_{\lambda}\left|C_{\lambda}\right|^{2} \delta\left(\omega-\omega_{\lambda}\right) / \rho(\omega)
\end{gathered}
$$

and

$$
C_{\lambda}=\frac{1}{N} \sum_{m l} \sum_{\gamma} \frac{\partial \pi_{x y}^{l}}{\partial u_{m \gamma}}\left[e_{l \gamma}(\lambda)-e_{m \gamma}(\lambda)\right] .
$$

In writing the above equations we have assumed that we are interested in the depolarized component of the spectrum, $(\alpha, \beta)=(x, y)$. Considering that

$$
\sum_{m} \frac{\partial \pi_{x y}^{l}}{\partial u_{m \gamma}}=0
$$

equation (A7) may be cast in the form 


$$
C_{\lambda}=\frac{1}{N} \sum_{m l} \sum_{\gamma} \frac{\partial \pi_{x y}^{l}}{\partial u_{m \gamma}} e_{m \gamma}(\lambda)
$$

or

$$
C_{\lambda}=\sum_{m} \sum_{\gamma} p_{m \gamma} e_{m \gamma}(\lambda)
$$

where

$$
p_{m \gamma}=\frac{1}{N} \sum_{l} \frac{\partial \pi_{x y}^{l}}{\partial u_{m \gamma}}
$$

$C_{\lambda}$ in equation ( $\mathrm{A8}$ ) has the same structure as $d_{\lambda}$ in equation (3), so that the components of the initial vector $\mathbf{t}^{(0)}$ are $p_{i} \equiv p_{m \gamma}$. We note that from equation (A6) we obtain

$$
\frac{\rho(\omega) C(\omega)}{2 \omega}=\sum_{\lambda}\left|C_{\lambda}\right|^{2} \frac{\delta\left(\omega-\omega_{\lambda}\right)}{2 \omega}=\sum_{\lambda}\left|C_{\lambda}\right|^{2} \delta\left(\omega^{2}-\omega_{\lambda}^{2}\right)
$$

to be compared with equation (2) in the text.

In this paper we have considered different mechanisms of polarizability modulation, i.e. different expressions for $\pi_{\alpha \beta}^{l}$, that result in different $\mathbf{t}^{(0)}$ 's. In particular, the explicit expressions for the studied scattering mechanisms are:

$$
\begin{aligned}
\text { DID : } & \pi_{\alpha \beta}^{l}=\sum_{m} \mathbf{T}_{\alpha \beta}^{(2)}(l m) \alpha_{l} \alpha_{m} \\
\text { NNDID : } & \pi_{\alpha \beta}^{l}=\sum_{m,\{l\}} \mathbf{T}_{\alpha \beta}^{(2)}(l m) \alpha_{l} \alpha_{m} \\
\text { BPOL : } & \pi_{\alpha \beta}^{l}=\sum_{m,\{l\}} \mathbf{T}_{\alpha \beta}^{(2)}(l m) V_{l m}
\end{aligned}
$$

where $\alpha_{l}$ is the bare polarizability of atom $l, \mathbf{T}_{\alpha \beta}^{(2)}(\mathbf{r})$ is the dipole propagator

$$
\mathbf{T}_{\alpha_{1} \alpha_{2} . . \alpha_{n}}^{(n)}(\mathbf{r})=\nabla_{1} \nabla_{2} \ldots \nabla_{n} \frac{1}{|\mathbf{r}|}
$$

the index $m,\{l\}$ indicates that the summation concerns all the $m$ nearest neighbors of atom $l$, and $V_{l m}$ is 1 or 0 according to whether atoms $l$ e $m$ are connected by a bond.

Therefore, from equation (A10) we find that:

$$
\text { DID : } \quad p_{i}=\sum_{l} \mathbf{T}_{x y \gamma}^{(3)}(l m) \alpha_{l} \alpha_{m}
$$




$$
\begin{aligned}
\text { NNDID : } & p_{i}=\sum_{l,\{m\}} \mathbf{T}_{x y \gamma}^{(3)}(l m) \alpha_{l} \alpha_{m} \\
\text { BPOL : } & p_{i}=\sum_{l,\{m\}} \mathbf{T}_{x y \gamma}^{(3)}(l m) V_{l m}
\end{aligned}
$$

In the case of DID the effective polarizability depends on the positions of all atoms, each weighted by the factor $1 / R^{3}$; in the NNDID case this effect is limited to nearest neighbors, to simulate induction mechanisms that decay faster than $1 / R^{3}$. In the case of BPOL modulation of polarizability occurs only if the nearest neighbors are actually connected by a bond. Therefore, in site percolators NNDID and BPOL coincide, while they do not in bond percolators.

We have also been interested in the calculation of the density of states; in this case the values of $p_{i}$ are uniformly and randomly distributed between -0.5 and 0.5 , as shown in ref. [1]. 


\section{REFERENCES}

[1] C. Benoit, E. Royer and G. Poussigue, J. Phys. Cond. Matt. 4, 3125 (1992), and references therein.

[2] C. Benoit, J. Phys. Cond. Matt. 1, 335 (1989).

[3] S. Alexander and R. Orbach, J. Phys. (Paris) Lett. 43, L625 (1982).

[4] R. Rammal and G. Toulouse, J. Phys. (Paris) Lett. 44, L13 (1983).

[5] M. Montagna, O. Pilla, G. Viliani, V. Mazzacurati, G. Ruocco and G. Signorelli, Phys. Rev. Lett. 65, 1136 (1990).

[6] V. Mazzacurati, M. Montagna, O. Pilla, G. Viliani, G. Ruocco, G. Signorelli, Phys. Rev. B 45, 2126 (1992).

[7] E. Stoll, M. Kolb and E. Courtens, Phys. Rev. Lett. 68, 2472 (1992).

[8] T. Nakayama, K. Yakubo and R. Orbach, Rev. Mod. Phys. 66, 381 (1994).

[9] P. Turchi, F. Ducastelle and G. Treglia, J. Phys. C 15, 2891 (1982).

[10] The density of states of large percolators at and above threshold, showing the typical crossover from phonon-like to fracton-like behavior, was early computed by E. Royer, C. Benoit and G. Poussigue, J. Phys. Cond. Matt. 4, 561 (1992). We reach a lower frequency, presumably thanks to the use of more moments.

[11] A. Aharony and D. Stauffer, Phys. Rev. Lett. 52, 2368 (1984).

[12] The average minimum frequency of the percolators scales as $\omega_{\min }(L)=c L^{-D / \bar{d}}$; the constant $c$ was determined by averaging the minimum eigenvalues obtained by diagonalizing the dynamical matrix of clusters with $L=30,40,50$ in $2 \mathrm{D}$, and $L=10,15,20$ in $3 \mathrm{D}$. In this way we obtain the values $c_{2}=4.5 \pm 0.2$ in $2 \mathrm{D}$ and $c_{3}=3.6 \pm 0.1$ in $3 \mathrm{D}$. These values of $c$ result in the following ratios $R_{d}(L)=\omega_{\min } / \omega_{\max }: R_{2}(650) \approx 1.6 \times 10^{-4}$ in $2 \mathrm{D}$, and $R_{3}(80) \approx 5 \times 10^{-4}$ in $3 \mathrm{D}$. However, in the first decade the levels are so sep- 
arate in a log-log plot that it is meaningless to speak about the density of states. It is interesting to follow the behavior of the spectrum in this frequency range with higher resolution than in Fig. $1\left(\varepsilon=10^{-9}\right)$ and with different numbers of moments. The lowest frequency peak in the density of states shifts to low frequency by more than one order of magnitude as the number of moments is increased from 1000 to 10,000, and at the same time the number of peaks in the reduced frequency decade $10^{-4} \div 10^{-3}$ increases from 1 to about 10. The spectrum becomes smooth in the next decade.

[13] G. H. Golub and C. F. Van Loan, Matrix Computations (Johns Hopkins University Press, Baltimore, 1989), Eq. (9.1.3), p. 478.

[14] W. A. Bowers and H. B. Rosenstock, J. Chem. Phys. 18, 1056 (1950).

[15] A. Boukenter, B. Champagnon, E. Duval, J. Dumas, J. F. Quinson and J. Serughetti, Phys. Rev. Lett. 57, 2391 (1986).

[16] E. Duval, G. Mariotto, M. Montagna, O. Pilla, G. Viliani and M. Barland, Europhys. Lett. 3, 333 (1987)

[17] G. Mariotto, M. Montagna, G. Viliani, R. Campostrini and G. Carturan, J. Phys. C 21, L797 (1988)

[18] Y. Tsujimi, E. Courtens, J. Pelous and R. Vacher, Phys. Rev. Lett. 60, 2757 (1988).

[19] S. Alexander, E. Courtens and R. Vacher, Physica A 195, 286 (1993).

[20] T. Keyes and T. Ohtsuki, Phys. Rev. Lett. 59, 603 (1987).

[21] A. Fontana, F. Rocca and M. P. Fontana, Phys. Rev. Lett. 58, 503 (1987).

[22] T. Achibat, A. Boukenter, and E. Duval J. Chem Phys. 99, 2046 (1993); and E. Duval, N. Garcia, T. Achibat, A. Boukenter, and J. Serughetti, ibidem 99, 2040 (1993).

[23] In Ref. [5], Fig. 3, DID and DID and NNDID are reported. 
[24] The calculation for bond percolators requires more computer memory, and this is the reason for the smaller linear dimensions.

[25] It should be stressed that removal of dangling bonds does not produce what is known as the backbone of the percolator.

[26] G. S. Grest and I. Webman, J. Phys. (Paris) Lett. 45, L1155 (1984).

[27] According to the analysis of ref. [19], though $\sigma>1$ is consistent with scaling, a "strong self similarity assumption would really imply $\sigma=1$ even for a fractal". 


\section{FIGURES}

FIG. 1. Log-log density of states of 2D site percolators (average of 10 realizations) having linear dimension $L=650$, with $\varepsilon=2 \cdot 10^{-6}$ and: (a) 5000 moments; (b) 1000 moments; (c) 50 moments. $\omega=\omega / \omega_{\max } ; m$ is the fitted slope; the curves are shifted vertically for graphical convenience.

FIG. 2. Scalar product $\left(\mathbf{t}^{(0)}, \mathbf{t}^{(n)}\right.$ ) (see text) as a function of the number of iterations $n$ for ordered square lattices of linear sizes 50 (a) and 100 (b), and 2D site percolators at threshold of linear sizes $650(\mathrm{c})$ and $50(\mathrm{~d})$.

FIG. 3. $C(\omega)$ for $2 \mathrm{D}$ site percolators, $L=650$. (a) DID; (b) BPOL. Average of 10 realizations.

FIG. 4. $C(\omega)$ for 3D site percolators, $L=80$. (a) DID; (b) BPOL. Average of 20 realizations. Open circles: DID for 3D site percolators, $L=40$, average of 100 realizations.

FIG. 5. $C(\omega)$ for 2D bond percolators, $L=500$. (a) DID; (b) BPOL. Average of 10 realizations.

FIG. 6. $C(\omega)$ for 3D bond percolators, $L=70$. (a) DID; (b) BPOL. Average of 10 realizations.

FIG. 7. Effect of electrical disorder on $C(\omega)$ for $2 \mathrm{D}$ site percolators, $L=650$. (a) $\alpha_{1}=0, \alpha_{2}=2$; (b) $\alpha_{1}=0.5, \alpha_{2}=1.5$; (c) $\alpha_{1}=\alpha_{2}=1$. Average of 10 realizations.

FIG. 8. $C(\omega)$ for $3 \mathrm{D}$ site percolators, $L=70$, above percolation threshold $(c=0.5)$. (a) Density of states; (b) DID; (c) BPOL; the curves are vertically shifted for graphical convenience. Average of 10 realizations 


\section{TABLES}

TABLE I. Fitted slopes, $m$, for $C(\omega)$, see Figures (3) to (8), and values of $\sigma$ obtained by fitting the numerical data with equations (15) and (16) for DID and NNDID respectively. For BPOL no such theoretical expression is available, but in the case of site percolators BPOL coincides with NNDID.

\begin{tabular}{|c|c|c|c|c|c|c|}
\hline & & \multicolumn{2}{|c|}{ DID } & \multicolumn{2}{|c|}{ NNDID } & \multirow{2}{*}{$\frac{\mathrm{BPOL}}{m}$} \\
\hline & & $m$ & $\sigma$ & $m$ & $\sigma$ & \\
\hline site & $2 \mathrm{D}$ & $0.80 \pm 0.02$ & $1.40 \pm 0.01$ & $1.24 \pm 0.01$ & $0.89 \pm 0.01$ & $1.24 \pm 0.01$ \\
\hline percolator & $3 \mathrm{D}$ & $0.41 \pm 0.03$ & $0.97 \pm 0.03$ & $1.56 \pm 0.01$ & $1.40 \pm 0.01$ & $1.56 \pm 0.01$ \\
\hline bond & $2 \mathrm{D}$ & $0.40 \pm 0.05$ & $1.12 \pm 0.04$ & $0.40 \pm 0.05$ & $0.30 \pm 0.05$ & $1.26 \pm 0.01$ \\
\hline percolator & $3 \mathrm{D}$ & $0.30 \pm 0.08$ & $0.86 \pm 0.07$ & $0.30 \pm 0.08$ & $0.30 \pm 0.05$ & $1.58 \pm 0.01$ \\
\hline
\end{tabular}

